\title{
Genetics of sleep disordered breathing (syndromes excluded)
}

\section{S. Plancoulaine}

INSERM, UMR1153, Center of research in epidemiology and statistics, Sorbonne University Paris Cité (CRESS), Early origins of child health and development team (ORCHAD), 94807 Villejuif, France; Paris-Descartes University, France

\section{SUMMARY}

The objective of this article is to present the approach, the main methods and the main results obtained in the study of the genetics of sleep disordered breathing. It is not an exhaustive systematic review of the literature but a didactic presentation to aid understanding of current research in the domain. It highlights the existence of a genetic predisposition to developing respiratory sleep disorder, from simple snoring to sleep apnea syndrome. The genes and pathways most frequently identified are those involved in inflammation.

\section{KEYWORDS}

Sleep disordered breathing, OSAHS, genetic predisposition, risk factors, inflammation

\section{INTRODUCTION}

There is a continuum of sleep disordered breathing, from snoring to apnea. A large part of the population are affected, increasingly with age and overweight/obesity, and more frequently males (20\%) than females $(10 \%)^{1}$. Although mostly studied in adults, respiratory sleep disorder also affects children (prevalence, $1 \%$ to $4 \%)^{2}$.

Sleep disordered breathing consists in iterative partial or complete obstruction of the upper airway ${ }^{3}$. Definitions and diagnostic criteria have evolved over time, but are still founded on polysomnography (PSG) or respiratory polygraphy $(\mathrm{RP})$ and the respiratory disturbance index (RDI). In epidemiology, these are seldom available, and studies have, so far, been mainly based on questionnaires and/or sleep diaries.

Several steps are needed to analyze genetic predisposition to a phenotypic trait, such as sleep disordered breathing, in humans. The first is to demonstrate a familial component: i.e., that certain families show abnormal numbers of cases. This is achieved by familial aggregation studies. The second is to demonstrate a genetic component within this familial component. This is mainly achieved by twin studies. When the phenotype is a common one las in the present case), genetic study uses genetic epidemiology, based on large samples (of families, of populations of several hundreds), using statistical methods, exploring for variations in markers or common genetic polymorphisms exerting moderate effects. The term here is "complex genetic predisposition" rather than Mendelian genetic predisposition, which identifiers genetic mutations or abnormalities that are generally rare but exert a strong deleterious effect, with an equally rare but severe phenotype (e.g., achondroplasia). 
The study of complex genetic predisposition has progressed since the 1990s, with the development of typing methods (of genes or gene expression), which have become increasingly available, technologically, methodologically and financially (Fig. 1). For about 10 years, study of gene expression (messenger RNA (mRNA)

\section{FAMILIAL COMPONENT}

\section{Familial aggregation}

There have been several reports of families with high incidence of sleep disordered breathing over several generations.

For example, El Bayadi et al., in the United States in 1990, reported a family of 9 spanning 3 generations recruited via an index case, a 55 yearold patient with severe snoring and apnea (apnea/hypopnea index (AHI) > 30/h), non-restorative sleep and excessive daytime somnolence ${ }^{4}$. Nine of the index case's siblings, children and grandchildren were habitual snorers with non-restorative sleep, 5 showed daytime somnolence, and 3 snored and showed sleep apnea. None showed retrognathia; 3 had a 22-32 mm mandibular plane - hyoid bone distance $(\mathrm{MPH}) ; 4$ had 7-9 $\mathrm{mm}$ pharyngeal airway space (PAS); and 5 were overweight (BMI, $\left.25-30 \mathrm{~kg} / \mathrm{m}^{2}\right)$. On RP, AHI was 15-30 in 4 cases, 5-15 in 3, including the grandson, and normal in only 1. This study thus shows familial aggregation of severe snoring, independently of BMI and more or less independently of cephalometry. measurement) and epigenetics (transcriptional modification without modification of DNA) or genome-wide association studies (GWAS) and/or direct whole-genome sequencing have been developing. Very recently, studies of networks or systems combining several such "omic" analyses have begun to be published.
In 1995, Guilleminault et al. reported on 3 American families of 3 generations, with children aged 3-16 years, diagnosed with sleep apnea syndrome (OSAS) confirmed on PSG and $\mathrm{RDI}>5 / h^{5}$. Sixteen of the 27 subjects examined and explored were diagnosed with obstructive sleep apnea syndrome (OSAS), including 7 children aged 3-16 years. Clinical examination found deep ogival palate and retropositioned mandible suggesting cephalometric abnormality.

Sundquist et al., in a siblings study, assessed the risk of hospital admission for OSAS in Swedish adults aged 19-72 years when an elder sibling had been admitted for OSAS ${ }^{6}$. A total of 12,763 male and 3,037 female subjects were included from Swedish registries. Standardized incidence ratios were calculated, distinguishing men and women, and, overall, proved on average ratios to be increased by a factor of just over 3 , regardless of gender.

They also found two peaks for age at diagnosis in male or female patients with familial history of OSAS 


\section{Complex genetic predisposition}

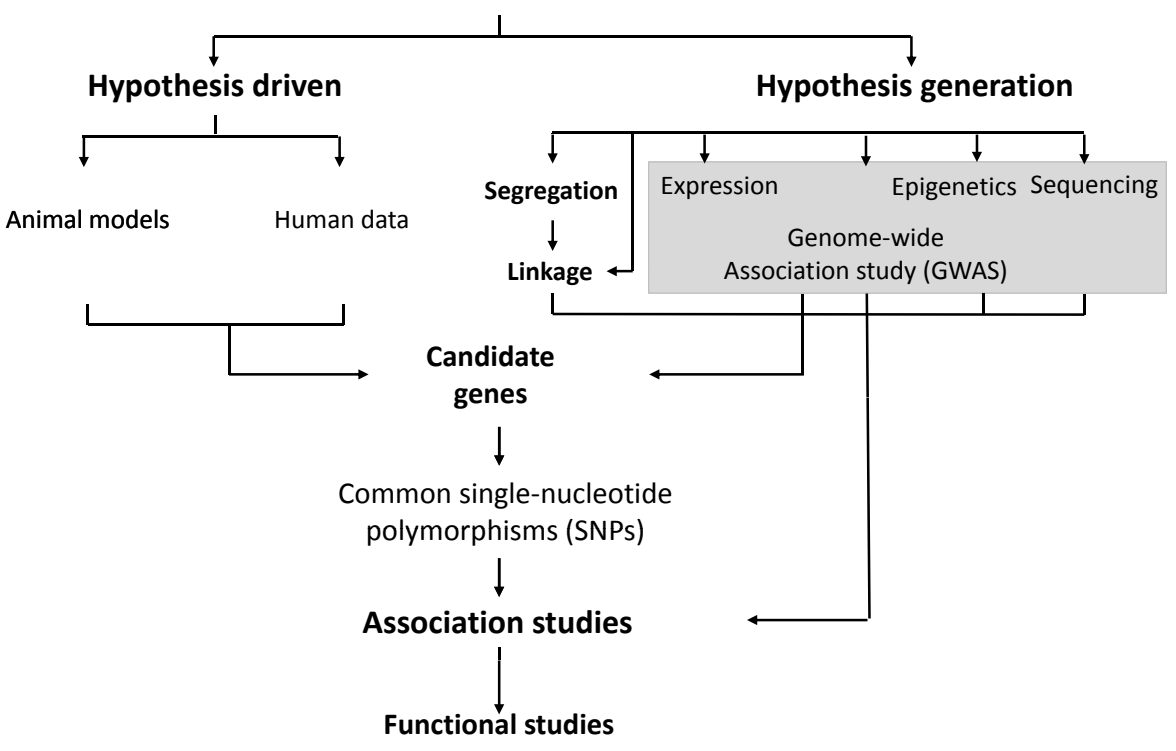

Figure 1

Flowchart of complex genetics methods according to presence of underlying hypothesis or not.

compared to those without: at 30-34 and 60-64 years, both more pronounced in men than women. They found no correlation between spouses, suggesting little impact of adult environment. Given the origin of the data, no clinical details were available (notably, BMI).

These studies thus show familial aggregation of respiratory sleep disorder, involving several generations, including children.

Some also suggest an impact of facial development and BMI, although without explaining all of the cases described. Such familial aggregation may represent a shared environmental component and/or a genetic component.

\section{Twin studies}

It is generally agreed that, for monozygotic twins (identical twins who devel- oped from a single ovum), phenotypic concordance is due to shared genetic inheritance. In dizygotic twins, who developed from two separate ova, phenotypic concordance is due only to the half of the genotype they share. Presence of a genetic component is suggested when phenotypic concordance is stronger in mono- than di-zygotic twins.

In the USA, Carmelli et al. (2001) studied 1,560 pairs of male twins with a mean age of 74 years and found $24 \%$ concordance for habitual snoring in monozygotic twins (818 pairs) compared to only $9 \%$ in dizygotic twins (742 pairs) ${ }^{7}$. Likewise, Desai et al., in the UK in 2004, studying 1,937 pairs of female twins with a mean age of 51 years (range, $20-80$ years), found $28 \%$ concordance for snoring disturbing the sleep of others and/or awakening them frequently or systematically in monozygotic twins (933 pairs) versus 
$14 \%$ in dizygotic twins $(1,004 \text { pairs })^{8}$.

In a more refined exploration of the twin pairs, Carmelli et al. focused on the coexistence of habitual snoring and obesity versus presence of only one or the other phenotype in their twin population, $29 \%$ of whom had BMI of at least $28 \mathrm{~kg} / \mathrm{m}^{2}$. They found $13 \%$ correlation between obesity and snoring, entirely attributable to shared genetic factors. They further estimated that only $30 \%$ of the genetic component in snoring could be attributed to genes also underlying obesity. Individual heritability of snoring was thus estimated at 23\% (95\% confidence interval (Cl): $18 \%, 28 \%$ ).

They thus suggested that snoring in elderly males has a familial basis with a genetic component that is largely independent of genes related to

\section{FAMILY STUDIES}

Historically, the first analyses of genetic predisposition were family studies: (i) familial segregation studies seeking to identify a so-called major gene with Mendelian transmission (dominant, co-dominant or recessive) within multigenerational families, or (ii) linkage studies seeking to locate a genetic effect, either on models derived from segregation analyses of multigenerational families or directly from data, usually concerning siblings, collected without preconception regarding the underlying genetic model of transmission.

\section{Segregation analyses}

The principle consists in comparing the distribution of the observed phenotype (sleep disordered breath- obesity?. Likewise, Desai et al., modeling a genetic effect as co-dominant with an environmental effect in their female twin pairs, estimated raw heritability of disturbing snoring at 52\% (95\% Cl:36\%, 68\%), falling to $42 \%$ after adjustment on BMI, smoking and menopausal status ${ }^{8}$.

Taken together, these studies demonstrate multigenerational familial aggregation of sleep disordered breathing, in different countries: i.e., in populations of differing origins (Caucasian, African American or Asian). They further demonstrated that this familial component was partly accounted for by genetic factors, and estimated heritability at $21 \%$ to $84 \%$ depending on population, phenotype (type of disorder studied) and associated factors such as BMI or morphology.

ing) in the studied population versus the expected distribution according to various Mendelian models (dominant, co-dominant or recessive), taking account of known risk factors (gender, BMI, etc.), familial resemblance (parent-child, sib-sib, etc.) and the characteristics of the major gene being modeled (frequency of "at-risk" allele, and probability of being affected according to genotype: i.e., penetrance). These analyses are purely statistical and require no genetic typing. Two such studies have been published.

Holberg et al. studied snoring using a self-administered questionnaire between 1972 and 1996 in 584 families totaling 2,019 non-Hispanic Caucasian individuals aged 10 years and 
over $^{9}$. They focused on two phenomena: isolated snoring, and snoring associated with daytime somnolence as proxy for obstructive sleep apnea/ hypopnea syndrome (OSAHS). They also took account of gender, age and $\mathrm{BMI}$ as risk factors. In isolated snoring, they found a significant familial resemblance between mothers and children and between siblings but not between spouses who present no genetic heritage in common. They found no major gene effect, and suggested a polygenic effect with several genes involved, none of which predominated, and/or shared environmental effects. In OSAHS, they found significant resemblances between parents and daughters, especially as regards mothers, pointing to a major dominant or co-dominant gene (either being compatible) with residual familial correlations suggestive of other unidentified genetic and/ or environmental effects.

Buxbaum et al. studied 177 Caucasian families (1,195 individuals) and 125 African American families (720 individuals), with ages between 2 and 85 years, from the Cleveland Familial Study cohort $(1990-1993)^{10}$. They focused on $\mathrm{AHI}$ measured at home by $\mathrm{RP}$, defined as at least 10 seconds' reduction in or arrest of airflow and at least $2.5 \%$ reduction in oxygen saturation. They found familial resemblance between $1^{\text {st }}$ degree relatives (parentchild, siblings) but not spouses, thus suggesting a genetic effect. In Caucasians, they found a recessive major gene after adjustment on age, explaining $28 \%$ of the variance. Adjustment on age and BMI eliminated the genetic effect in males, suggesting a major role of BMI. In African
American families, they found a codominant major gene, accounting for $35 \%$ of variance; adjustment on BMI enhanced this genetic effect.

These two studies demonstrated genetic heterogeneity: several genes seem to be involved, some exerting a Mendelian effect (major genes), the genes and associated factors such as BMI varying according to population.

\section{Linkage analysis}

Once a genetic model has been established, the major gene has to be located within the genome. This is achieved by genetic linkage analysis, using parameters estimated on segregation analysis. Linkage analysis involves typing genetic polymorphisms using dedicated or non-dedicated chips, and thus requires DNA from family members. One or more regions of the genome are then explored for genes which are transmitted within the family in the same way as the gene characterized by the parameters estimated on segregation analysis. Linkage is measured by LOD (logarithm of odds) score. No such studies have been published regarding snoring or OSAHS.

Another approach involves linkage analysis without presuppositions as to the genetic model underlying transmission. In the case of pairs of siblings, for example, for each polymorphism, the proportion of shared alleles that are identical by descent (IBD: identical to those of the parents) is compared to the proportion expected on Mendelian grounds (Fig. 2): there is a genetic relation when there is an excess of IBD alleles shared by siblings presenting 


\section{\begin{tabular}{|l|l|l|l|l|l|}
\hline$A B$ & $C D$ \\
\hline
\end{tabular}

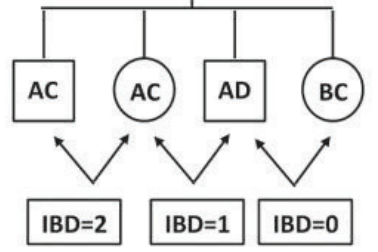 \\ $\rightarrow$ There is genetic linkage when there is an excess of IBD alleles shared by affected siblings \\ Based on shared parental alleles identical by descent (IBD) \\ Expected distribution for pair of siblings

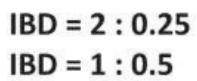

Figure 2

Diagram of linkage analysis without presuppositions on the underlying genetic model of transmission, based on sibling pairs.

the same phenotype. Palmer et al. and Larkin et al. performed such analyses in the above-mentioned Cleveland population ${ }^{11-13}$. They obtained genotyping data for 641 Caucasians (109 families) and 634 African Americans (128 families). They identified several regions with LOD scores suggesting linkage with $\mathrm{AHI}$ and/or OSAHS. In Caucasians, the main peaks for $\mathrm{AHI}$ were on chromosomes 6 and 10 in 6q23-25 and 10q24-q25, respectively. These two peaks, which diminish after adjustment for BMI, suggest a pleiotropic effect of these regions. Another promising region was in 6p11-q11, close to the orexin-2 receptor gene, suggesting passage via a cascade independent of BMI. In African Americans, the strongest links with $\mathrm{AHI}$ after adjustment on BMI were in 8p21.3, increasing after adjustment on BMI, and in 8q24.1, decreasing after adjustment on BMI. These analyses suggest that there are genetic regions related to sleep disordered breathing, acting both independently of BMI and by pathways linked to $\mathrm{BMI}^{11}$.

Relf et al. performed linkage analysis in a Filipino family of 50 (50\% adults), recruited via a child presenting with OSAHS diagnosed by PSG ${ }^{14}$. The family showed high prevalence of OSAHS and metabolic abnormalities: $46 \%$ of members (9 adults, 14 children) had OSAHS, severe in 5 cases (4 adults, 1 child). Moreover, at least two metabolic abnormalities (obesity, type-2 diabetes, hypertriglyceridemia, insulin resistance, or HDL or total cholesterol elevation) were found in $48 \%$ of the children. Linkage analysis found a significant peak in 19q13.4 for both OSAHS and HDL cholesterol level. Several candidate genes are present in this linkage region, including NK cell immunoglobulin receptor genes involved in modulating inflammatory response to cellular stress and to onset of atheroma.

The next step comprises casecontrol or association studies on candidate genes identified within the linkage regions. 


\section{CASE-CONTROL STUDIES}

\section{Probable candidate genes}

Case-control studies on probable candidate genes compare polymorphism frequency in candidate genes identified on linkage analysis or by prior knowledge found in the literature. To the best of our knowledge, no case-control studies have been published following the above-mentioned linkage studies. On the other hand, there have been several based on the literature, focusing on genes involved in inflammation and/or immunity ${ }^{15-22}$, metabolism $15-17,23-25$, cardiovascular disease ${ }^{16-18,26-28}$, or neurological and sleep disorder ${ }^{16,19-21,29-32}$. Figure 3 shows the overlap between some of these fields.

\section{GWAS}

For about the last 10 years, genotyping techniques and costs have greatly changed, allowing wholegenome case-control studies, known as Genome-Wide Association Studies: GWASs. In sleep disordered breathing, a consortium was set up to centralize data on Caucasians and African Americans: the CARe consortium. A dedicated chip, comprising genes targeting the heart, lungs, blood and sleep, was used, including slightly more than 45,000 polymorphisms. As the study included several populations from different cohorts, a metaanalysis approach was adopted. Two phenotypes were focused on: AHI

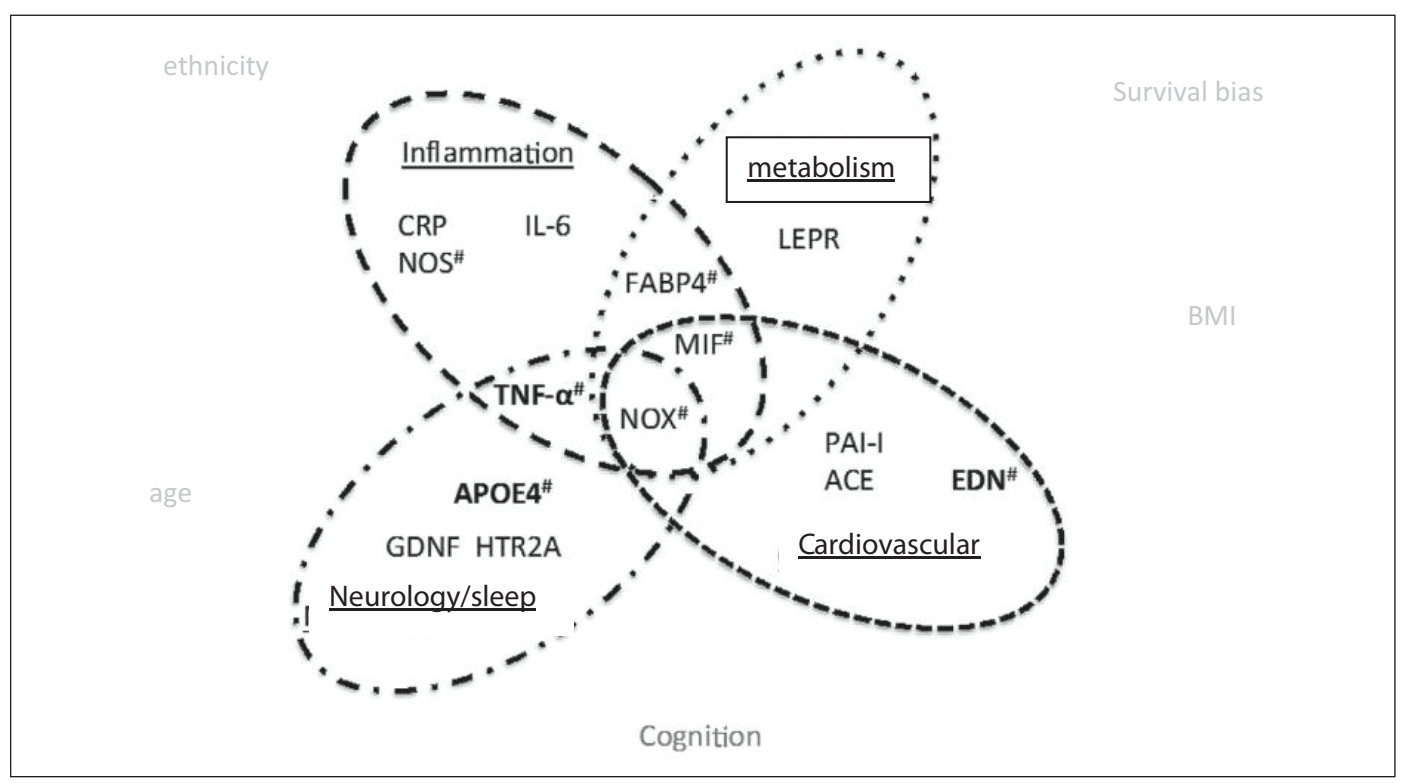

Figure 3

Main genes associated with sleep disorder breathing in case-control studies. In bold, genes replicated by different teams. \#: results for both adults and children. In grey, associated factors taken into account or not according to the study. 
and OSAS diagnosed on PSG or RP $(A H I \geq 15 / h)$. For Caucasians, the main analysis concerned 2,904 individuals (664 from the Cleveland study, 1,673 from the Atherosclerosis Risk in the Community (ARIC) study, and 567 from the Framingham study ${ }^{33}$ ).

For $\mathrm{AHI}$, there were no significant findings (with $p<10^{-6}$ ). However, the polymorphism with the smallest $p$-value lay on chromosome 9 in a non-coding region of the LPAR 1 gene, which codes for a protein with a pro-inflammatory role promoting circulating monocytes ${ }^{34}$ and also known to be associated with neurologic and craniofacial abnormalities in mice $^{35}$. Regarding OSAHS, analysis revealed a significantly associated polymorphism on chromosome 1 in a non-coding region of PTGER3, with enhanced association after adjustment on BMI. PTGER3 codes for a protein modulating central and peripheral neurotransmitter release. It is also part of a haplotype (group of polymorphisms transmitted together) associated with onset of high blood pressure $^{36}$. The polymorphism with the next lowest $p$-value was the one observed on chromosome 9 and associated with the $\mathrm{AHI}$ phenotype.

For African Americans, the main analysis concerned 647 individuals from the Cleveland cohort. Analysis found a significant association ( $p<$ $10^{-6}$ ) between $\mathrm{AHI}$ and the chromosome 9 polymorphism in the noncoding region of $L P A R 1$, enhanced in $\mathrm{n}$ on-obese subjects, and an almost significant association with a polymorphism in a non-coding region of ITPR2, the corresponding protein playing a role in intracellular calcium regulation and being implicated in inflam- mation, endothelial dysfunction and blood-pressure abnormalities ${ }^{37}$. There was also a significant negative association in non-obese subjects between OSAHS and a polymorphism lying close to the PLEK gene, coding for a protein involved in actin assembly within leukocytes and thus contributing to exocytosis. The third polymorphism identified, although non-significant, concerned the LPAR1 gene on chromosome 9.

The next step was to replicate these results in similar populations on a case-control study of candidate polymorphisms. Three were selected, but only two could be adequately typed (LPAR1 on chromosome 9, and PTGER3 on chromosome 1), in 1,795 Caucasians taken from the Western Australia Sleep Health Study (WASHS) and 1,010 African Americans taken from the Cleveland study ( $n=459$ ) and the Case Transdisciplinary Research in Energetics and Cancer Colon Polyps Study (CTRECCPS) ( $\mathrm{n}=551)$. These replication populations differed from the primary populations in terms of prevalence of OSAHS: the Caucasian population included more males (63\% versus 47\%) and obese subjects (mean BMI, 33 versus $29 \mathrm{~kg} / \mathrm{m}^{2}$ ), with a higher prevalence of OSAHS (77\% versus 33\%); the African American population was composed of two very different populations with female predominance (78\% and $67 \%)$, one being a female OSAHS cohort with BMI of 41 versus $32 \mathrm{~kg} / \mathrm{m}^{2}$ (n = 459), and the other being a female cohort with BMI of $31 \mathrm{~kg} / \mathrm{m} 2$, free of sleep disordered breathing $(n=551)$. The association between $\mathrm{AHI}$ and the PTGER3 polymorphism on chromosome 1 on a co-dominant model was 
replicated in Caucasians, as well as the association between OSAHS and the LPAR1 polymorphism on chromosome 9 in African Americans. These two findings confirm that a complex genetic predisposition is involved in sleep disordered breathing. The two polymorphisms concern genes implicated in inflammation, raising the question of causality. Moreover, taking BMI into account seems to make little or no difference, suggesting that the effects are independent. Functional studies will be needed to establish the causal role of these polymorphisms and to understand the mechanisms involved, but have yet to be performed and/or published.

\section{Gene expression (messenger RNA)}

\section{Studies based on candidate gene transcription (with prior knowledge)}

A recent functional study by David Gozal's team ${ }^{18}$ included 605 children aged 5-10 years, with or without OSAHS, and concerned polymorphisms of a candidate gene, EDN1, coding for a protein involved in an intracellular signal implicated in vasoconstriction, left ventricle hypertrophy and asthma ${ }^{38,39}$. It was recently shown that four EDN1 polymorphisms are frequent in children with OSAHS. The functional study showed that EDN1 expression, assessed by cell quantity of specific messenger RNA (mRNA), was higher in children with OSAHS than in those without (mean, 1.8 versus 1.65).

\section{Studies based on genome-wide transcription (without prior knowledge)}

Another approach is to explore whole-genome transcription, as was also done by David Gozal's team ${ }^{40}$ in a case-control study in 40 nonobese children aged 4-10 years, the 20 case subjects having OSAHS on PSG, with tonsillar hypertrophy. The chip, containing 44,000 transcripts (mRNA) covering the whole genome, identified 68 transcripts from white blood cells with differential (over or under) expression in cases. They were thus able to identify biological cascades or pathways, including 23 cellular and 32 functional processes, among which inflammatory response predominated. These methods open up complementary lines of research on the mechanisms underlying biological and organic abnormalities, but require molecular and functional confirmation.

\section{Epigenetics}

Gene expression may be altered without change in the genetic sequence itself. Several mechanisms have been identified that increase or decrease gene expression: (i) histone modification: histones are protein groups which chromosomal DNA curls around; modification may consist in acetylation, methylation, phosphorylation or ubiquitination; (ii) direct DNA methylation; or (ii) micro-mRNA fixing on DNA. DNA methylation has been most widely studied.

David Gozal's team performed a first case-control study in 5-10 yearolds matched for age, gender and ethnicity, with or without OSAHS on PSG $^{41}$. They studied DNA methylation in a candidate gene (FOXP3) and its regulatory regions. The coded protein is involved in immune regulation ${ }^{42}$. They demonstrated hypermethylation 
in the FOXP3 promoter region, especially in case of CRP elevation, which is a marker for inflammation. They demonstrated hypermethylation of the eNOS promoter region in 4-12 year-olds with OSAHS associated with delayed post-occlusion response ${ }^{43}$, inducing significant reduction in mRNA expression as compared to children without response delay, proportionally to post-occlusion response time.

\section{Expression-based networks and systems}

Information from several "omic" studies can be combined, allowing global network or system analysis, based on bioinformatics. One example is Liu et al.'s study ${ }^{44}$, in which a network was created from candidate genes and/or proteins identified in the literature, taking account of genes and/or proteins involved in craniofacial morphology, obesity,

\section{CONCLUSIONS/PERSPECTIVES}

Research into the genetics of sleep disordered breathing is progressing. Results so far demonstrate a complex genetic predisposition and point to involvement of specific genes according to phenotype (e.g., $\mathrm{AHI}$ or OSAHS, or isolated or associated OSAHS), ethnicity, presence of overweight/obesity, etc. Even so, most genes playing a role in whatever context seem to be part of pro-inflammatory cascades. The reports require confirmation, by replication or functional or other studies. inflammation, respiratory control and pleiotropic effects. Networking was weighted by the number of genes and proteins included and their inter-relations. Data from public data-bases on protein-protein interactions and adipose tissue sequencing were then included, along with gene expression data (relative mRNA quantification) from a case-control study of postoperative subcutaneous and visceral adipose tissue from adult patients with or without OSAHS. Frequency scores were then calculated for each protein according to the networks they belong to, ranking them in decreasing order. The most frequent proteins, whatever the network, were those mainly involved in inflammation, some of which had already been associated with OSAHS (e.g., PDGF, EDN1, NOS3) or associated disorders (e.g., PI3K, STAT, MAPK, associated with insulin resistance, inflammation, etc.).

However, to this end there remain many questions in suspense and methodological issues to resolve. Which phenotypes should be studied? Using what definitions? Should the focus be on early and/or severe forms, or take account of a fuller range of factors and associated disorders (BMI, craniofacial development, etc.)? Which populations should be studied? Specific sub-populations: children, at-risk ethnic groups? What sample size? Sample size is very important if "omic" methods are used, 
so as to ensure sufficient detection power despite multiple testing. But how is it to be achieved? Should cohorts be shared in a consortium? If so, how to deal with heterogeneity of populations (gender, age, ethnicity) and of definitions of phenotypes of interest: identical diagnostic criteria across cohorts? Should the same complementary data be collected, using identical definitions and coding: BMI, craniofacial data, etc.? Are the genetic, epigenetic and protein typing methods identical or at least partially superimposable? There are indeed many chips on the market, and they are developing rapidly. Finally, what type of analysis is needed? Adjusted, stratified, matched? On which criteria? Should gene-gene and gene-environment interactions be studied? Should several genes be studied simultaneously (e.g., haplotypes)? Should approaches be combined, especially "omics"? What methods? What criteria?

A lot of work, then, remains to be done to shed light on the pathophysiology of sleep disordered breathing, and perhaps improve management.

Conflicts of interest: The authors declare no conflicts of interest.

\section{REFERENCES}

1. Peppard PE, Young T, Barnet JH, Palta M, Hagen EW, Hla KM. Increased Prevalence of Sleep-Disordered Breathing in Adults. Am J Epidemiol 2013;177(9):1006-14.

2. Nespoli L, Caprioglio A, Brunetti L, Nosetti L. Obstructive sleep apnea syndrome in childhood. Early Hum Dev 2013;89:S33-7.

3. American Academy of Sleep Medicine. International classification of sleep disorders. 3rd ed. Darien, IL, USA: American Academy of Sleep Medicine, 2014.

4. El Bayadi S, et al. A family study of sleep apnea. Anatomic and physiologic interactions. Chest 1990;98(3):554-9.

5. Guilleminault C, Partinen M, Hollman K, Powell N, Stoohs R. Familial aggregates in obstructive sleep apnea syndrome. Chest 1995;107(6):1545-51.

6. Sundquist J, Li X, Friberg D, Hemminki K, Sundquist K. Obstructive sleep apnea syndrome in siblings: an 8-year Swedish follow-up study. Sleep 2008;31(6):817-23.

7. Carmelli D, Bliwise DL, Swan GE, Reed T. Genetic factors in self-reported snoring and excessive daytime sleepiness: a twin study. Am J Resp Crit Care Med 2001;164(6): 949-52.

8. Desai AV, Cherkas LF, Spector TD, Williams AJ. Genetic influences in self-reported symptoms of obstructive sleep apnoea and restless legs: a twin study. Twin Res 2004;7(6):589-95.

9. Holberg CJ, Natrajan S, Cline MG, Quan SF. Familial Aggregation and Segregation Analysis of Snoring and Symptoms of Obstructive Sleep Apnea. Sleep Breath 2000;4(1): 21-30.

10. Buxbaum SG, Elston RC, Tishler PV, Redline S. Genetics of the apnea hypopnea index in Caucasians and African Americans: I. Segregation analysis. Genet Epidemiol 2002;22(3):243-53.

11. Larkin EK, Patel SR, Elston RC, Gray-McGuire C, Zhu X, Redline S. Using Linkage Analysis to Identify Quantitative Trait Loci for Sleep Apnea in Relationship to Body Mass Index. Ann Hum Genet 2008;72(6):762-73. 
12. Palmer LJ, et al. Whole Genome Scan for Obstructive Sleep Apnea and Obesity in African-American Families. Am J Resp Crit Care Med 2004;169(12):1314-21.

13. Palmer $L J$, et al. A whole-genome scan for obstructive sleep apnea and obesity. Am J Hum Genet 2003;72(2):340-50.

14. Relf BL, Larkin EK, Torres CD, Baur LA, Christodoulou J, Waters KA. Genome-wide linkage of obstructive sleep apnoea and high-density lipoprotein cholesterol in a Filipino family: bivariate linkage analysis of obstructive sleep apnoea: Obstructive sleep apnoea genome scan. J Sleep Res 2010;19(2):349-57.

15. Bhushan $B$, et al. Fatty-acid binding protein 4 gene polymorphisms and plasma levels in children with obstructive sleep apnea. Sleep Med 2011;12(7):666-71.

16. Gozal D, Khalyfa A, Capdevila OS, Kheirandish-Gozal L, Khalyfa AA, Kim J. Cognitive function in prepubertal children with obstructive sleep apnea: A Modifying Role for NADPH Oxidase p22 Subunit Gene Polymorphisms? Antioxid Redox Signal 2012;16(2):171-7.

17. Khalyfa A, Kheirandish-Gozal L, Capdevila OS, Bhattacharjee R, Gozal D. Macrophage migration inhibitory factor gene polymorphisms and plasma levels in children with obstructive sleep apnea. Pediatr Pulmonol 2012;47(10):1001-11.

18. Chatsuriyawong $S$, et al. Polymorphisms in nitric oxide synthase and endothelin genes among children with obstructive sleep apnea. BMC Med Genom 2013;6(1):29.

19. Khalyfa A, Serpero LD, Kheirandish-Gozal L, Capdevila OS, Gozal D. TNF-othelin genes among children with factor gene polymorphisms and pic Obstructive Sleep Apnea. J Pediatr 2011;158(1):77-82.

20. Huang J, Liao N, Huang Q-P, Xie Z-F. Association between tumor necrosis factor- $\alpha-$ 308G/A polymorphism and obstructive sleep apnea: a meta-analysis. Genet Test Mol Biomarkers 2012;16(4):246-51.

21. Larkin EK, et al. A Candidate Gene Study of Obstructive Sleep Apnea in European Americans and African Americans. Am J Resp Crit Care Med 2010;182(7):947-53.

22. Larkin EK, et al. A Study of The relationship between the Interleukin-6 Gene and obstructive sleep apnea. Clin Transl Sci 2010;3(6):337-9.

23. Popko K, et al. Frequency of distribution of leptin receptor gene polymorphism in obstructive sleep apnea patients. J Physiol Pharmacol 2007;58(5):551-61.

24. Hanaoka M, Yu X, Urushihata K, Ota M, Fujimoto K, Kubo K. Leptin and leptin receptor gene polymorphisms in obstructive sleep apnea syndrome. Chest 2008;133(1):79-85.

25. Kalra M, Chakraborty R. Leptin and Leptin Receptor Gene Polymorphisms in Obstructive Sleep Apnea Syndrome Response. Chest 2008;133(6):1530-1.

26. Patel SR, Larkin EK, Mignot E, Lin L, Redline S. The association of angiotensin converting enzyme (ACE) polymorphisms with sleep apnea and hypertension. Sleep 2007;30(4):531-3.

27. Mehra R, et al. Sleep-disordered Breathing and Prothrombotic Biomarkers: Cross-Sectional Results of the Cleveland Family Study. Am J Resp Crit Care Med 2010;182(6):82633.

28. Barcel6:826):826Sleep-disordered Breathing and Prothrombotic Biomarkers: CrossSectional Results of the Cleveland Family Study.s with obstructive sleep apnoea. Resp Med 2002;96(3):193-6.

29. Gislason T, Pack Al, Helgadottir HT, Stefansson K, Besenbacher S, Jonsdottir I. The CRP and GDNF genes do not contribute to apnea-hypopnea index or risk of obstructive sleep apnea. Am J Resp Crit Care Med 2011;184(1):143-4.

30. Gottlieb DJ, et al. APOE epsilon4 is associated with obstructive sleep apnea/hypopnea The Sleep Heart Health Study. Neurology 2004;63(4): 664-8. 
31. Kadotani $\mathrm{H}$, et al. Association between apolipoprotein E epsilon4 and sleep-disordered breathing in adults. JAMA 2001;285(22):2888-90.

32. Kalra $M$, et al. Association of ApoE genetic variants with obstructive sleep apnea in children. Sleep Med 2008;9(3):260-5.

33. Patel SR, et al. Association of Genetic Loci with Sleep Apnea in European Americans and African-Americans: The Candidate Gene Association Resource (CARe). PLoS ONE 2012;7(11):e48836.

34. Maugeri N, et al. LPAR1 and ITGA4 regulate peripheral blood monocyte counts. Hum Mutat 2011;32(8):873-6.

35. Contos JJ, Fukushima N, Weiner JA, Kaushal D, Chun J. Requirement for the IpA1 lysophosphatidic acid receptor gene in normal suckling behavior. Proc Natl Acad Sci USA 2000;97(24):13384-9.

36. Sõber S, et al. Targeting 160 Candidate genes for Blood Pressure Regulation with a Genome-Wide Genotyping Array. PLoS ONE 2009;4(6):e6034.

37. Wilker $\mathrm{EH}$, et al. Candidate genes for respiratory disease associated with markers of inflammation and endothelial dysfunction in elderly men. Atherosclerosis 2009;206(2):480-5.

38. Kaoukis A, et al. The role of endothelin system in cardiovascular disease and the potential therapeutic perspectives of its inhibition. Curr Top Med Chem 2013;13(2):95-114.

39. Goldie RG, Fernandes LB. A possible mediator role for endothelin-1 in respiratory disease. Monaldi Arch Chest Dis 2000;55(2):162-7.

40. Khalyfa A, Capdevila OS, Buazza MO, Serpero LD, Kheirandish-Gozal L, Gozal D. Genome-wide gene expression profiling in children with non-obese obstructive sleep apnea. Sleep Med 2009;10(1):75-86.

41. Kim J, et al. DNA Methylation in Inflammatory Genes among Children with Obstructive Sleep Apnea. Am J Resp Crit Care Med 1 2011;185(3):330-8.

42. Devaud C, Darcy PK, Kershaw MH. Foxp3 expression in T regulatory cells and other cell lineages. Cancer Immunol Immunother 2014;63(9):869-76.

43. Kheirandish-Gozal L, Khalyfa A, Gozal D, Bhattacharjee R, Wang Y. Endothelial Dysfunction in Children With Obstructive Sleep Apnea Is Associated With Epigenetic Changes in the eNOS GeneDNA Methylation amp Endothelial Dysfunction in Apnea. Chest 2013;143(4):971-7.

44. LiuY, et al. Systems Biology Analyses of Gene Expression and Genome Wide Association Study Data in Obstructive Sleep Apnea. Pac Symp Biocomput 2011:14-25. 